\title{
Why Sliding Friction of Ne and Kr Monolayers Is So Different on the $\mathrm{Pb}(111)$ Surface
}

\author{
Y. N. Zhang, ${ }^{1}$ F. Hanke, ${ }^{2}$ V. Bortolani, ${ }^{3}$ M. Persson, ${ }^{2,4}$ and R. Q. Wu ${ }^{1}$ \\ ${ }^{1}$ Department of Physics and Astronomy, University of California, Irvine, California, 92697 USA \\ ${ }^{2}$ The Surface Science Research Center, The University of Liverpool, Liverpool, L69 3BX, United Kingdom \\ ${ }^{3}$ Dipartimento di Fisica e CNISM, Università di Modena e Reggio Emilia, Via Campi 213/A, Modena, 41100 Italy \\ ${ }^{4}$ Department of Applied Physics, Chalmers University of Technology, SE-412 96, Göteborg, Sweden \\ (Received 14 December 2010; revised manuscript received 11 May 2011; published 8 June 2011)
}

To understand the tribological properties of $\mathrm{Ne}$ and $\mathrm{Kr}$ on $\mathrm{Pb}(111)$, the potential energy surfaces for sliding motion of $\mathrm{Ne}, \mathrm{Kr}$, and $\mathrm{Xe}$ monolayers on the $\mathrm{Pb}(111)$ surface are examined through density functional calculations, using either local density or self-consistent nonlocal van der Waals functionals. The calculated adsorption energy for $\mathrm{Xe} / \mathrm{Pb}(111)$ agrees well with experiment, validating the present approach and parameters. Activation energies along a sliding path indicate that Ne motion is much faster than $\mathrm{Kr}$ and $\mathrm{Xe}$ on $\mathrm{Pb}(111)$ at $T \sim 6 \mathrm{~K}$, which explains the puzzling experimental observation.

DOI: 10.1103/PhysRevLett.106.236103

PACS numbers: 68.35.Af, 68.43.Bc, 71.15.Nc

The continuing advances in surface physics make it now possible to study tribological properties of various materials at the atomic scale and to reach an unprecedented level of understanding on the origin of friction [1]. Recent measurements performed with apparatuses such as quartz crystal microbalance (QCM) $[2,3]$ and atomic force microscopy [4,5] established that the friction laws at the nanoscale are different from the macroscopic Amontons laws. Intriguing and sometimes puzzling experimental observations have stimulated new theoretical studies of friction, aiming at the development of clear microscopic understandings on friction and wear. The difficulties in testing hypotheses are known to be a major theoretical problem. While a tremendous number of parameters may affect the theoretical results of lateral forces between two solids, macroscopic measurements only provide a single number: the friction coefficient. This situation made the validation or falsification of theories practically impossible. The advent of controlled atomic scale measurements substantially changed the situation and new computational techniques on the other hand enable theorists to study models that include the relevant internal degrees of freedom of the contacting surfaces. Indeed, theoretical studies [6-10] of friction have touched on various effects such as electron-hole pair excitations, chemical reactions, vibration relaxations, and the dynamics of defect creation and diffusion.

Because of the weak interaction across interfaces, systems with adsorption of rare-gas ( $R G$ ) atoms on solid surfaces are ideal to tackle tribological issues at the atomic level. The $\mathrm{Pb}(111)$ surface was frequently used as a substrate since it is possible to deposit several layers of $\mathrm{Pb}$ on the quartz electrode in UHV for the convenience of QCM measurements. Furthermore, surface morphologies measured with scanning tunneling microscopy indicate the presence of wide atomic terraces with a size of about $50 \mathrm{~nm}$ on $\mathrm{Pb}(111)$ [11], much larger than the typical
QCM oscillating amplitude of $\sim 5 \mathrm{~nm}$. One striking finding in this context is the drastic difference between the sliding friction of $\mathrm{Ne}$ and $\mathrm{Kr}$ mono- or multilayers [12]. While it was shown that one or a few monolayers of Ne slide on $\mathrm{Pb}$ (111) and a slip time is measurable ( $~ 3$ nanoseconds), $\mathrm{Kr}$ layers are pinned (slip time is essentially zero) at low temperature $(\sim 6.5 \mathrm{~K})$. The present study is devoted to the microscopic explanation of this unusual behavior. We have calculated the potential energy surfaces for $\mathrm{Ne}, \mathrm{Kr}$ and $\mathrm{Xe}$ monolayers on $\mathrm{Pb}(111)$. Indeed, the $\mathrm{Pb}(111)$ surface is found to be substantially "rougher" for $\mathrm{Kr}$ than for $\mathrm{Ne}$, as manifested by significantly different activation energies for their sliding motion, in good agreement with experimental observations.

To simulate $\mathrm{RG} / \mathrm{Pb}(111)$, we used a periodic model with a seven-layer slab for $\mathrm{Pb}(111)$, a monolayer of RG adatoms, and a $\sim 20 \AA$ thick vacuum region. As depicted in Fig. 1(a), a $(\sqrt{3} \times \sqrt{3}) R 30^{\circ}$ supercell was adopted. This cell is the simplest commensurate structure for RG monolayers on close-packed metal surfaces, as revealed by LEED measurements $[13,14]$, and also as used in previous theoretical studies [15]. Density functional theory calculations were performed using the Vienna $a b$ initio simulation package (VASP) [16] along with projector augmented wave pseudopotentials [17]. An $(8 \times 8 \times 1)$ MonkhorstPack $k$-point grid was used to sample the Brillouin zone and an energy cutoff of $400 \mathrm{eV}$ was used for the basis expansion. We fully optimized positions of adatoms and the top five $\mathrm{Pb}$ layers and fixed the two bottommost $\mathrm{Pb}$ layers at their bulk positions.

Recent ab initio calculations indicated that the localdensity approximation (LDA) and generalized-gradient approximation [18] gave inconsistent descriptions for the structural properties of RG adsorption on metal surfaces, and LDA was found to yield better results $[19,20]$. Nevertheless, one needs to be cautious in dealing with sliding problems of RG since the energetics might be 

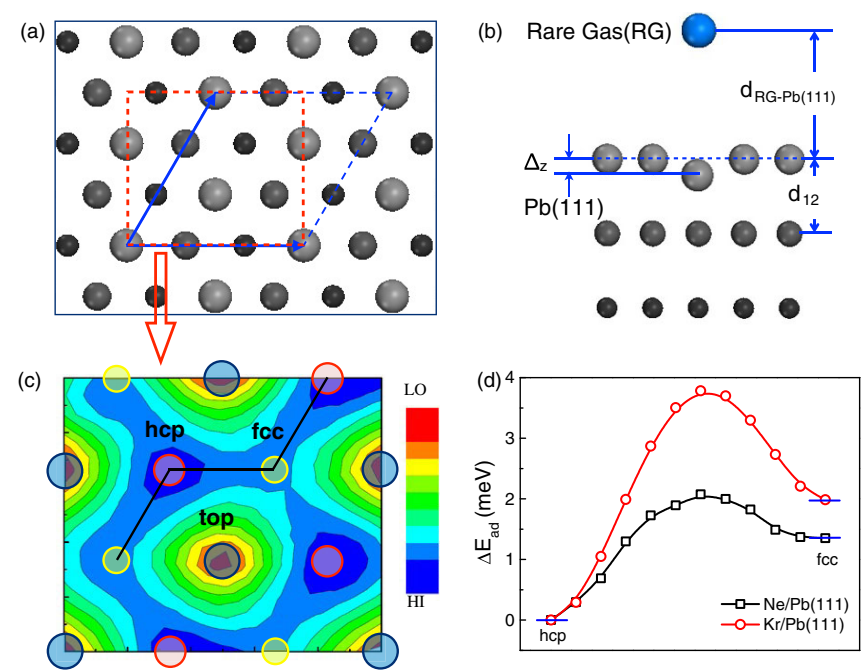

FIG. 1 (color online). Panels (a) and (b) are top and side views of the atomic model adopted in the present calculations. The parallelogram and rectangle in (a) show the $\sqrt{3} \times \sqrt{3}$ unit cell, and the "mapping" region displayed in panel (c), respectively. The large, medium, and small spheres are for the surface, subsurface, and third layer $\mathrm{Pb}$ atoms and notations $d_{\mathrm{RG}-\mathrm{Pb}(111)}$ and $\Delta_{z}$ are marked in for the convenience of discussions. Panels (c) and (d) show results for $\mathrm{Ne} / \mathrm{Pb}(111)$ using LDA: contours of $E_{\mathrm{ad}}$ and changes of $E_{\mathrm{ad}}$ along the path hcp $\rightarrow$ bridge $\rightarrow$ fcc, respectively.

affected by the van der Waals (vdW) interactions that are missing in traditional density functional calculations [21-23]. In the present studies, we first treated the exchange-correlation effect at the level of LDA and, furthermore, investigated the influence of $\mathrm{vdW}$ interactions using the nonlocal energy functional (vdW-DF) proposed by Langreth and Lundqvist [24] as implemented selfconsistently in VASP by Klimeš, Bowler, and Michaelides [25]. As an exchange term, we used the OPTB86B version [25], which gives a lattice constant of $4.98 \AA$ for the bulk $\mathrm{Pb}$, in close agreement with experiments, when including the $5 d$ shell of $\mathrm{Pb}$ as valence electrons in the projector augmented wave. As a benchmark for the studies of energetics, the calculated adsorption energy [defined as $E_{\mathrm{ad}}=E_{\mathrm{tot}}-E_{\mathrm{Pb}(111)}-E_{\mathrm{RG}-\mathrm{ML}}$ where $E_{\mathrm{tot}}, E_{\mathrm{Pb}(111)}$, and $E_{\mathrm{RG}-\mathrm{ML}}$ are total energies of $\mathrm{RG} / \mathrm{Pb}(111), \mathrm{Pb}(111)$, and the isolated RG monolayer, respectively] of $\mathrm{Xe} / \mathrm{Pb}(111)$ is $172.6 \mathrm{meV}$, close to the experimental value, $191 \pm 10 \mathrm{meV}$ [26].

Although friction depends on the effective dissipation of kinetic energies of atoms, the simplest picture would connect the sliding friction to the hopping probability of adatoms between adjacent stable and metastable sites. To quantitatively describe the "roughness" of $\mathrm{Pb}(111)$ toward the sliding motion of RG adatoms, we sampled the $\sqrt{3} \times \sqrt{3}$ supercell with about 40 mesh points in the real space, and calculated the position dependence of $E_{\mathrm{ad}}$. These data were used to construct potential energy
TABLE I. The adsorption energy, $E_{\text {ad }}(\mathrm{meV})$, and interlayer distance, $d_{\mathrm{RG}-\mathrm{Pb}(111)}(\AA)$, for symmetric structures of $\mathrm{Ne} / \mathrm{Pb}(111)$ and $\mathrm{Kr} / \mathrm{Pb}(111)$, obtained through LDA and vdW-DF (ОРТВ86B exchange) calculations. The activation energy, $E_{a}(\mathrm{meV})$, is also given for RG sliding motion.

\begin{tabular}{lrrrr}
\hline \hline & \multicolumn{2}{c}{$\mathrm{Ne}$} & \multicolumn{2}{c}{$\mathrm{Kr}$} \\
& LDA & vdW-DF & \multicolumn{1}{c}{ LDA } & vdW-DF \\
\hline$E_{\mathrm{ad}}^{\text {atop }}$ & -36.3 & -46.8 & -82.1 & -125.1 \\
$E_{\mathrm{ad}}^{\mathrm{fcc}}$ & -42.7 & -51.3 & -95.0 & -133.9 \\
$E_{\mathrm{ad}}^{\text {hcp }}$ & -44.1 & -51.6 & -97.0 & -134.9 \\
$d_{\mathrm{RG}-\mathrm{Pb}(111)}^{\text {atop }}$ & 3.3 & 3.8 & 3.7 & 3.9 \\
$d_{\mathrm{RG}-\mathrm{Pb}(111)}^{\text {hc,fcc }}$ & 3.0 & 3.5 & 3.5 & 3.8 \\
$E_{a}$ & 2.1 & 0.7 & 3.8 & 2.5 \\
\hline \hline
\end{tabular}

surfaces, as displayed in Fig. 1(c) for $\mathrm{Ne} / \mathrm{Pb}(111)$ from the LDA calculations. According to results for highsymmetric geometries in Table I, both $\mathrm{Ne}$ and $\mathrm{Kr}$ prefer the high-coordination hcp-hollow site, and disfavor the low-coordination atop site. The energy differences between the atop and hcp adsorption geometries within LDA are $7.8 \mathrm{meV}$ and $14.9 \mathrm{meV}$ for $\mathrm{Ne} / \mathrm{Pb}(111)$ and $\mathrm{Kr} / \mathrm{Pb}(111)$, respectively. The corresponding values obtained within the vdW-DF are somewhat smaller: 4.8 and $9.8 \mathrm{meV}$ for $\mathrm{Ne}$ and $\mathrm{Kr}$ on $\mathrm{Pb}(111)$.

It is significant to note in Table I that the inclusion of $\mathrm{vdW}$ interactions does not alter the site preference for $\mathrm{Ne}$ and $\mathrm{Kr}$, despite the noticeable changes of bond lengths and adsorption energies. To better appreciate this result, LDA and vdW-DF adsorption energies are plotted in Fig. 2 against the interplanar distance for $\mathrm{Ne}$ over the highsymmetric sites of $\mathrm{Pb}(111)$. While the potential wells are deepened in vdW-DF compared to LDA, Ne and $\mathrm{Kr}$ both still favor the hcp site. Meanwhile, the distances,

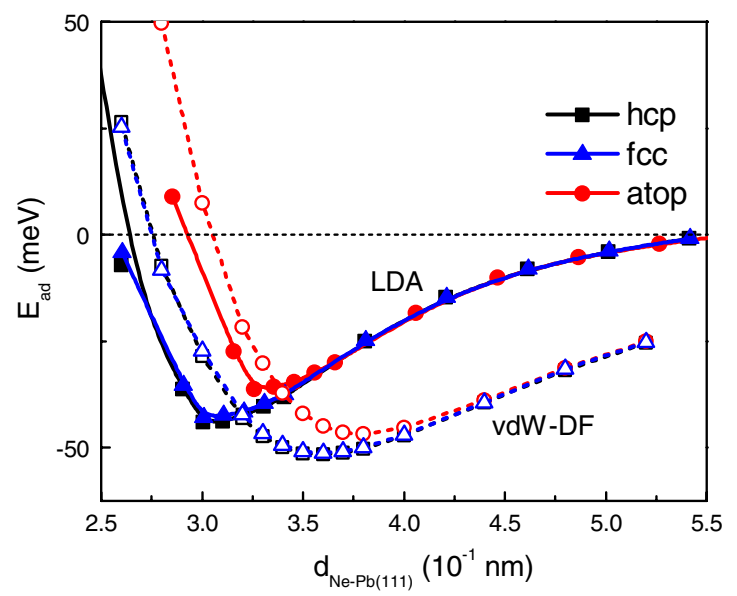

FIG. 2 (color online). The position and distance dependent adsorption energies of $\mathrm{Ne} / \mathrm{Pb}(111)$. Solid and open symbols represent results from the LDA and vdW-DF using the OPTB86B exchange term, respectively. 
$d_{\mathrm{RG}-\mathrm{Pb}(111)}$, expand by $\sim 0.2-0.5 \AA$ from the LDA data. The induced relaxations on $\mathrm{Pb}(111), \Delta_{z}$ in Fig. 1(b), are smaller than $0.01 \AA$.

The contours of $E_{\text {ad }}$ in Fig. 1(c) show that RG atoms slide on $\mathrm{Pb}(111)$ along a "zigzag" route as highlighted by the lines, hcp $\rightarrow$ bridge $\rightarrow$ fcc $\rightarrow$ bridge $\rightarrow$ hcp, under the influence of lateral pushing forces. To appreciate the difference between $\mathrm{Ne}$ and $\mathrm{Kr}$, we present their results of $\Delta E_{\text {ad }}=E_{\text {ad }}^{\text {site }}-E_{\text {ad }}^{\text {hcp }}$ along the path hcp $\rightarrow$ bridge $\rightarrow$ fcc in Fig. 1(d). The activation energies, $E_{a}$, can be directly calculated from the change of $E_{\mathrm{ad}}$ between the ground state and transition state, which occurs over the bridge site for both $\mathrm{Ne}$ and $\mathrm{Kr}$ adatoms. It is striking to see that values of $E_{a}$ are very different: $2.1(0.7) \mathrm{meV}$ for $\mathrm{Ne}$ versus 3.8 (2.5) $\mathrm{meV}$ for $\mathrm{Kr}$ through LDA (vdW-DF) calculations. Using the Arrhenius equation with $E_{a, \mathrm{Kr}}-E_{a, \mathrm{Ne}}=$ $1.7 \mathrm{meV}$ (LDA) or $1.8 \mathrm{meV}$ (vdW-DF) and $T=6.5 \mathrm{~K}$, one can estimate that $\mathrm{Kr}$ hopping should be more than 20 times slower than $\mathrm{Ne}$, even without considering the mass effect of $\mathrm{Kr}$ on the frequency factor. Obviously, the "unusual" difference between the sliding friction properties observed for $\mathrm{Ne}$ and $\mathrm{Kr}$ on $\mathrm{Pb}(111)$ mainly stems from the difference between their $E_{a}$ [12].

Now one may ask: "What makes the values of $E_{a}$ different for $\mathrm{Ne}$ and $\mathrm{Kr}$ ?" An important quantity for the understanding of the bonding mechanism is the electron density difference, $n(\boldsymbol{r})=n_{\mathrm{RG} / \mathrm{Pb}(111)}(\boldsymbol{r})-n_{\mathrm{Pb}(111)}(\boldsymbol{r})-n_{\mathrm{RG}-\mathrm{ML}}(\boldsymbol{r})$, obtained from electron densities of $\mathrm{RG} / \mathrm{Pb}(111), \mathrm{Pb}(111)$, and the isolated RG monolayer, respectively. Plots of $\Delta n(\boldsymbol{r})$ for $\mathrm{Ne} / \mathrm{Pb}(111)$ and $\mathrm{Kr} / \mathrm{Pb}(111)$ in Fig. 3 show that electrons of RG adatoms obviously polarize toward the substrate, in consistence with previous studies of RG adsorptions on metal surfaces [20]. The contrasts between $\mathrm{Ne}$ and $\mathrm{Kr}$ manifest themselves through (1) the magnitude of $\Delta n(\boldsymbol{r})$ of $\mathrm{Ne} / \mathrm{Pb}(111)$ being notably weaker than that of $\mathrm{Kr} / \mathrm{Pb}(111)$, (2) the influence of $\mathrm{Kr}$ extending to interior $\mathrm{Pb}$ layers, whereas $\mathrm{Ne}$ appears to only affect the top $\mathrm{Pb}$ (a) $\mathrm{Ne} / \mathrm{Pb}(111)$

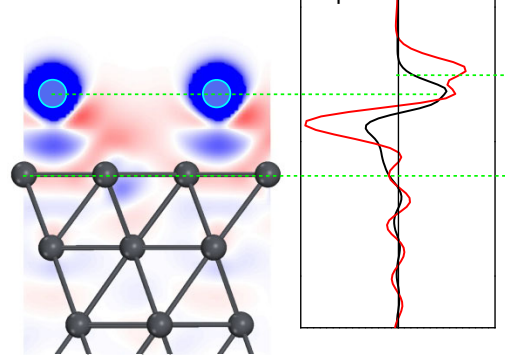

(b) $\mathrm{Kr} / \mathrm{Pb}(111)$

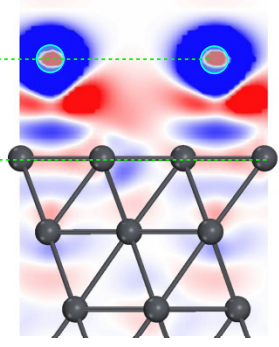

FIG. 3 (color online). Electron density differences of (a) $\mathrm{Ne}$ and (b) $\mathrm{Kr}$ on $\mathrm{Pb}(111)$ shown in the $(11 \overline{2})$ plane within the range of $\pm 8 \times 10^{-5} e / \AA^{3}$. Red (light gray) and blue (dark gray) represent electron accumulation and depletion, respectively. Line profiles in the middle show the planar averages of $\Delta n(\mathbf{r})$ along the surface normal, with the black line for $\mathrm{Ne} / \mathrm{Pb}(111)$ and red (gray) line for $\mathrm{Kr} / \mathrm{Pb}(111)$. layer. It is more clear from the $\Delta n(z)$ curves in the middle panel in Fig. 3, the planar averages of $\Delta n(\boldsymbol{r})$ along the vertical direction, that the amplitude of electron polarization in $\mathrm{Kr} / \mathrm{Pb}(111)$ is about 2 times larger than that in $\mathrm{Ne} / \mathrm{Pb}(111)$. The disturbance of $\mathrm{Ne}$ fades quickly below the surface $\mathrm{Pb}$ layer, in contrast to the oscillating $\Delta n(z)$ of $\mathrm{Kr}$ in this region. While the stronger charge polarization of $\mathrm{Kr}$ leads to larger $E_{\mathrm{ad}}$, the range of disturbance is crucial for the distinction between $\mathrm{Kr}$ and $\mathrm{Ne}$ in their $E_{a}$ as well as $\Delta E_{\mathrm{ad}}$ between the fcc and hop sites.

More information can be unraveled from the partial density of states (PDOS) in Fig. 4. One may easily see a major dissimilarity between $\mathrm{Ne}$ and $\mathrm{Kr}$ : the $p$ states of $\mathrm{Ne}$ are located at $-9.7 \mathrm{eV}$ whereas those of $\mathrm{Kr}$ are located at $-5.6 \mathrm{eV}$. As shown by the shaded regions in Fig. 4(a), Pb has a large band gap between its $s$ and $p$ states above $-6.5 \mathrm{eV}$. Ne $p$ states overlap with $\mathrm{Pb} s$ states in energy and they have a weak hybridization in the energy range between $-10.2 \mathrm{eV}$ and $-8.2 \mathrm{eV}$. The $\mathrm{Kr} p$ states, on the other hand, are located right in the $\mathrm{Pb}$ band gap and thus the $\mathrm{Kr}-\mathrm{Pb}$ hybridization is weak. The topmost $\mathrm{Pb}$ atoms are slightly affected by RG adatoms, as demonstrated by curves of $\triangle \mathrm{DOS}$ in Fig. 4(b) $(\triangle \mathrm{DOS}=$ $\left.\mathrm{DOS}_{\mathrm{Pb} \text { in RG/Pb(111) }}-\mathrm{DOS}_{\mathrm{Pb} \text { in } \mathrm{Pb}(111)}\right)$. Some $\mathrm{Pb} s$ states

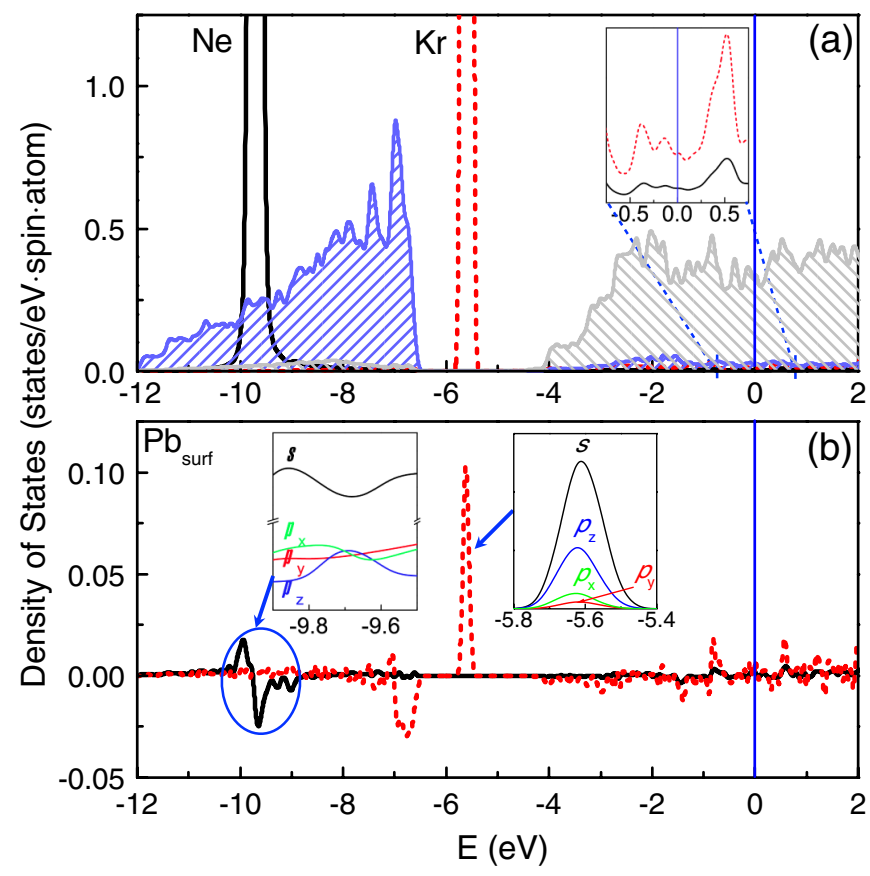

FIG. 4 (color online). (a) PDOS around $\mathrm{Ne}$ (solid line) and $\mathrm{Kr}$ (dashed line) on $\mathrm{Pb}(111)$; the inset displays the induced $p$ states of $\mathrm{Ne}$ and $\mathrm{Kr}$ around the $E_{F}$; the blue (dark gray) and light gray shaded regions give the $s$ and $p$ states of around the $\mathrm{Pb}$ atoms. (b) The RG-induced change in PDOS of the topmost $\mathrm{Pb}$ atom; the insets show contributions from different $s$ and $p$ partial waves around the $\mathrm{Pb}$ surface atom at $-9.7 \mathrm{eV}$ in $\mathrm{Ne} / \mathrm{Pb}(111)$ and at $-5.6 \mathrm{eV}$ in $\mathrm{Kr} / \mathrm{Pb}(111)$, respectively. The vertical lines indicate the position of the Fermi level. 
shift down in energy around $-9.7 \mathrm{eV}$, a signature of $\mathrm{Ne}-\mathrm{Pb}$ hybridization in $\mathrm{Ne} / \mathrm{Pb}(111)$. In the left-side inset of Fig. 4(b), one finds energy splittings between $p_{z}$ and $p_{x, y}$ states of Ne, due to the crystal field effect from the substrate. Strikingly, $\mathrm{Kr}$ produces a pronounced peak at $-5.6 \mathrm{eV}$ in the $\triangle \mathrm{DOS}$ curve of $\mathrm{Pb}_{\text {surf }}$ in Fig. 4(b), as well as in the second and third $\mathrm{Pb}$ layers (not shown here). Further decomposition of this peak reveals its $s$ and $p_{z}$ contributions, as shown in the right-side inset of Fig. 4(b). It is clear from these DOS curves that RG-Pb interactions mainly occur in the low-energy region. Both DOS and $\triangle D O S$ of $\mathrm{Ne}$ and $\mathrm{Kr}$ are very small around the Fermi level in Figs. 4(a) and 4(b). Therefore, changes of $\mathrm{Pb}$ properties around $E_{F}$ (e.g., going through superconducting transition) affect the friction negligibly [27].

In summary, we performed LDA and self-consistent vdW-DF calculations to explain the unusual difference between the observed sliding friction of $\mathrm{Ne}$ and $\mathrm{Kr}$ monolayers on the $\mathrm{Pb}(111)$ surface. Ne and $\mathrm{Kr}$ prefer the hcphollow site in their equilibrium adsorption geometries and slide along a path over hcp $\rightarrow$ bridge $\rightarrow$ fcc $\rightarrow$ bridge $\rightarrow$ hcp sites. However, the activation energy of $\mathrm{Ne}$ is noticeably smaller than that of $\mathrm{Kr}$, which leads to a huge difference between their mobilities on $\mathrm{Pb}(111)$ at $6.5 \mathrm{~K}$. Analyses of electronic properties reveal substantial charge polarization from RG adatoms toward the substrate as well as orbital intermixing in the interfacial region. In particular, the long-range disturbance of $\mathrm{Kr}$ in $\mathrm{Pb}$ is identified as the key factor for the pinning of $\mathrm{Kr}$ on $\mathrm{Pb}(111)$ at low temperature. As a prediction, the calculated $E_{a}$ for the Xe monolayer sliding on $\mathrm{Pb}(111)$ is also large, $4.1 \mathrm{meV}$ with the LDA, and the Xe monolayer also should be pinned by the substrate at low temperature. The present work provides a valuable example of using density functional simulations for quantitative studies of friction at the nanoscale, via the direct determination of potential energy surfaces and activation energies.

This work was supported by the DOE-BES (Grant No. DE-FG02-05ER46237). Calculations were performed on parallel computers at NERSC and those provided by SNAC and VR in Sweden.

[1] B. N. J. Persson, Sliding Friction: Physical Principles and Applications (Springer, Berlin, 1998).

[2] J. Krim and A. Widom, Phys. Rev. B 38, 12184 (1988).

[3] J. Krim, D. H. Solina, and R. Chiarello, Phys. Rev. Lett. 66, 181 (1991).
[4] G. Binnig, C. F. Quate, and C. Gerber, Phys. Rev. Lett. 56, 930 (1986).

[5] C. M. Mate, G. M. McClelland, R. Erlandsson, and S. Chiang, Phys. Rev. Lett. 59, 1942 (1987).

[6] B. N. J. Persson, Phys. Rev. B 48, 18140 (1993).

[7] A. Franchini, M. Brigazzi, G. Santoro, and V. Bortolani, J. Phys. Condens. Matter 19, 305014 (2007); 20, 224019 (2008).

[8] Y. Mo, K. T. Turner, and I. Szlufarska, Nature (London) 457, 1116 (2009).

[9] V. Bortolani, A. Franchini, G. Santoro, and M. Brigazzi, Tribol. Lett. 39, 251 (2010).

[10] M. Persson, Solid State Commun. 80, 917 (1991).

[11] L. Bruschi, G. Fois, A. Pontarollo, G. Mistura, B. Torre, F. Buatier de Mongeot, C. Boragno, R. Buzio, and U. Valbusa, Phys. Rev. Lett. 96, 216101 (2006).

[12] L. Bruschi, M. Pierno, G. Fois, F. Ancilotto, G. Mistura, C. Boragno, F. Buatier de Mongeot, and U. Valbusa, Phys. Rev. B 81, 115419 (2010).

[13] M. Caragiu, Th. Seyller, and R. D. Diehl, Phys. Rev. B 66, 195411 (2002).

[14] C. Hückstädt, S. Schmidt, S. Hüfner, F. Forster, F. Reinert, and M. Springborg, Phys. Rev. B 73, 075409 (2006).

[15] J. L. F. Da Silva, C. Stampfl, and M. Scheffler, Phys. Rev. Lett. 90, 066104 (2003).

[16] G. Kresse and J. Furthmüller, Comput. Mater. Sci. 6, 15 (1996).

[17] P. E. Blöchl, Phys. Rev. B 50, 17953 (1994).

[18] J. P. Perdew, K. Burke, and M. Ernzerhof, Phys. Rev. Lett. 77, 3865 (1996).

[19] M. Petersen, S. Wilke, P. Ruggerone, B. Kohler, and M. Scheffler, Phys. Rev. Lett. 76, 995 (1996).

[20] J. L. F. Da Silva and C. Stampfl, Phys. Rev. B 77, 045401 (2008).

[21] P. Lazić, Ž. Crljen, R. Brako, and B. Gumhalter, Phys. Rev. B 72, 245407 (2005).

[22] M. Rohlfing and T. Bredow, Phys. Rev. Lett. 101, 266106 (2008).

[23] G. Román-Pérez and J. M. Soler, Phys. Rev. Lett. 103, 096102 (2009).

[24] M. Dion, H. Rydberg, E. Schröder, D. C. Langreth, and B. I. Lundqvist, Phys. Rev. Lett. 92, 246401 (2004); 95, 109902(E) (2005); D. C. Langreth et al., J. Phys. Condens. Matter 21, 084203 (2009).

[25] J. Klimeš, D. Bowler, and A. Michaelides, Phys. Rev. B 83, 195131 (2011).

[26] N. Ferralis, H. I. Li, K. J. Hanna, J. Stevens, H. Shin, F. M. Pan, and R. D. Diehl, J. Phys. Condens. Matter 19, 056011 (2007).

[27] M. Pierno, L. Bruschi, G. Fois, G. Mistura, C. Boragno, F. Buatier de Mongeot, and U. Valbusa, Phys. Rev. Lett. 105, 016102 (2010). 